Relations industrielles

Industrial Relations

Comment concevoir un système intégré de gestion, par P. J. Yvon et C. Semin, Paris, Entreprise Moderne d'Édition, 1970, 260 pp.

\title{
Laurent Bélanger
}

Volume 26, numéro 2, 1971

URI : https://id.erudit.org/iderudit/028237ar

DOI : https://doi.org/10.7202/028237ar

Aller au sommaire du numéro

\section{Éditeur(s)}

Département des relations industrielles de l'Université Laval

\section{ISSN}

0034-379X (imprimé)

1703-8138 (numérique)

Découvrir la revue

Citer ce compte rendu

Bélanger, L. (1971). Compte rendu de [Comment concevoir un système intégré de gestion, par P. J. Yvon et C. Semin, Paris, Entreprise Moderne d’Édition, 1970, 260 pp.] Relations industrielles / Industrial Relations, 26(2), 516-517.

https://doi.org/10.7202/028237ar

Tous droits réservés (C) Département des relations industrielles de l'Université Laval, 1971
Ce document est protégé par la loi sur le droit d'auteur. L'utilisation des services d'Érudit (y compris la reproduction) est assujettie à sa politique d'utilisation que vous pouvez consulter en ligne.

https://apropos.erudit.org/fr/usagers/politique-dutilisation/ 
section syndicale de l'entreprise et les attitudes nouvellement acquises de la maîtrise à l'endroit des pratiques à adopter dans ses relations avec les ouvriers et les employés. D'une part, les directions cherchent à garder jalousement leur autorité en maintenant à distance, en harcelant, en menaçant même les délégués de personnel et la section syndicale. D'autre part, la maîtrise sert de tampon où s'isole à la fois la direction et les ouvriers en cherchant, par un savoirvivre et un savoir-faire fraîchement acquis, à atténuer le poids et l'ommiprésence de l'autorité.

C'est là le type de contradictions auxquelles nous faisions allusion plus haut et qui semblent inhérents à un effort d'intégration des travailleurs dans l'entreprise. Chaque cas étudié révèle un degré d'intégration plutôt faible, plus marqué par le conflit ouvert ou larvé que par l'harmonie au sein des rapports sociaux. Au risque d'errer, nous croyons qu'un enseignement se dégage de cette étude, un enseignement que la plupart des directions ont tendance à oublier. Le développement d'un climat de relations interpersonnelles authentiques nous semble impossible dans une entreprise où la direction cherche à démolir l'institution syndicale ou refuse «d'avoir les coudées franches 》 avec les représentants des différentes catégories de personnel.

\section{Laurent BELANGER}

\section{De la prévision économique à la gestion}

financière, par Jacques Gervais, Paris, Entreprise Moderne d'Edition, 1970, $351 \mathrm{pp}$.

Dans ce volumineux ouvrage, l'auteur traite de l'influence de la prévision économique sur la gestion financière dans trois domaines qu'il assigne à la gestion financière: l'orientation générale des investissements; le calcul économique des solutions les plus avantageuses; le financement des investissements.

Dans chacun de ces domaines, son étude porte successivement sur la gestion financière de l'Etat, élément de l'environnement économique, et la gestion financière de l'entreprise, compte tenu de cet environnement.

Ce volume s'adresse d'abord et plus particulièrement à des lecteurs français car l'auteur désire, par son ouvrage, in- téresser et enseigner les cadres et les spécialistes financiers de France sur la gestion prévisionnelle, technique pour mieux orienter les investissements et la marche des entreprises de façon à atteindre le niveau de rentabilité que connaissent les entreprises américaines.

L'ouvrage est cependant d'intérêt pour quiconque s'intéresse à ces questions, et plus particulièrement aux techniques de l'analyse financière que l'auteur étudie longuement dans la deuxième partie. Les explications sont de telle nature qu'il n'est pas nécessaire d'être un spécialiste pour les aborder et en tirer profit, toutefois même un spécialiste pourra y retrouver ses notions théoriques de base et retracer l'élément qu'il avait oublié.

Jean-Pierre BEAULIEU

\section{Comment concevoir un système intégré de gestion, par P. J. Yvon et C. Semin, Paris, Entreprise Moderne d'Edition, $1970,260 \mathrm{pp}$.}

La plupart des ouvrages sur l'utilisation intensive et systématique de l'ordinateur en administration des entreprises sont en anglais. Les Entreprises modernes d'édition, avec le concours des auteurs P.J. Yvon et C. Semin, tous deux ingénieurs à Dubold-France, viennent de mettre sur le marché cet ouvrage en français sur les systèmes intégrés de gestion (Integrated Management Information System). C'est l'intégration en un seul système des applications, existantes et à concevoir, du traitement automatique des données à la gestion de l'entreprise.

Les deux auteurs en partant de leur expérience personnelle et des théories existantes ont dégagé les principes et les modalités de mise en œuuvre d'un système intégré.

Dans ce type de système, les données ne sont saisies qu'une seule fois pour être traitées automatiquement dans plusieurs programmes correspondant à des objectifs différents. En d'autres mots, des services ou des unités administratives différentes qui utilisent une information de base identique touchant l'accomplissement d'une activité devraient se concerter pour cueillir les données et les entrer dans l'ordinateur sans faire une duplication. Ce principe amène les auteurs à préciser la signification d'une 
expression nouvelle: la zone naturelle d'information (ZNI). En applicant ce principe à l'échelle de l'entreprise, on en arrive à la définition d'une autre expression : la base commune des données (BCD). Ces données communes à toute l'entreprise peuvent subir autant de traitements différents que l'exigent les services utilisateurs.

Les modalités de mise en cuvre du système intégré sont relativement faciles à saisir :

- Dans une première phase, on effectue un diagnostic de la nature de l'information habituellement utilisée dans la prise de décision et le contrôle à tous les niveaux.

- La deuxième phase consiste dans la détermination des zones naturelles d'information. C'est le regroupement des informations selon les fonctions.

- La mise sur pied d'un département d'informatique de même que l'inventaire de l'équipement nécessaire constituent la troisième phase.

- Vient ensuite la conception proprement dite d'un système intégré.

- Enfin, c'est la concrétisation du système et lancement de son exploitation.

C'est une étude qu'on peut lire avec profit, même si on n'est pas familier avec le language de l'ordinateur et la complexité des opérations qu'il peut effectuer.

Laurent BELANGER

Valeurs économiques et valeurs juridiques dans les fusions d'entreprises, par Philippe Comte, Paris. Entreprise Moderne d'Edition, 4, rue Cambon, 1970, 269 pages.

Depuis assez longtemps déjà, on assiste à un phénomène qui se généralise de plus en plus : la fusion ou le regroupement des entreprises. C'est ce phénomène qu'étudie Philippe Comte tant dans ses implications économiques que juridiques.

L'étude est divisée en trois parties: la première traite de ce que l'Auteur appelle « l'environnement économique des fusions d'entreprises 》; la deuxième est consacrée aux «valeurs économiques》 des fusions calculées en vue de la détermination des droits respectifs des actionnaires sur le nouvel ensemble; enfin, la troisième s'occupe des implications de nature juridique.

Comme il est facile de le deviner, les deux dernières parties de cet ouvrage ont un caractère technique marqué et s'adressent surtout aux spécialistes qui ont à analyser les obstacles qu'on peut rencontrer à l'occasion d'une fusion ainsi que les avantages qui peuvent en ressortir.

La première partie est d'une portée plus générale. L'auteur y étudie les causes générales des fusions et les obstacles qui s'y opposent, le rôle de la fusion d'entreprises parmi les modes de concentration à l'œuvre dans la société moderne, les conditions de réussite des fusions et l'attitude des pouvoirs publics.

André ROY

L'audiovisuel au service de la formation, méthodes-matériels, par Robinson $\mathbf{P}$. Rigg, Paris, Entreprise Moderne d'Edition, 1971, 220 pp.

Définitivement, les ouvrages de l'Entreprise moderne d'édition retiennent de plus en plus l'attention par leur qualité et la nature des sujets traités qui sont d'un grand intérêt pour ceux concernés par la gestion du personnel.

Le volume de R. P. Rigg originalement publié en anglais sous le titre Audiovisual Aids and Techniques in Managerial and Supervisory Training en 1969 est très bien traduit par José Ponzone.

Il faut dire dès maintenant que ce livre constitue presqu'une somme des techniques et des matériels de l'audiovisuel existant actuellement; ce qui se comprend assez bien étant donné d'une part, la nouveauté relative de cette aide pédagogique dans les procédés et méthodes de formation et, d'autre part, la compétence et l'expérience de l'auteur qui depuis 1945 a été mêlé à tout ce qui, de près ou de loin, concerne le mouvement audiovisuel dans les entreprises et les administrations en Grande-Bretagne.

L'auteur traite de six points principaux :

1. Le problème de la formation;

2. Du choix et de l'emploi des programmes de formation;

3. Les aides audiovisuelles et leurs utilisations ;

4. Techniques de présentation; 